\title{
The burden of silicosis, pulmonary tuberculosis and COPD among former Basotho goldminers
}

Authors: Brendan V Girdler-Brown ${ }^{\mathrm{a}}$, Neil W White ${ }^{\mathrm{b}}$, Rodney I Ehrlich ${ }^{\mathrm{c}}$, Gavin J

Churchyard $^{\mathrm{d}}$

\section{Author qualifications and affiliations:}

${ }^{a}$ MBChB, MMed, FCPHM(SA): School of Health Systems and Public Health, University of Pretoria, Pretoria, South Africa

${ }^{\mathrm{b}}$ MBChB, MMed, FCP(SA), MD: Department of Medicine, University of Cape Town, Cape Town, South Africa

${ }^{\mathrm{c}} \mathrm{MBChB}$, MFOM, FCPHM(SA), PhD: School of Public Health and Family Medicine, University of Cape Town, Cape Town, South Africa

${ }^{\mathrm{d}}$ MBBCh, FCP(SA) PhD: Aurum Institute for Health Research, Johannesburg, South Africa and Centre for the AIDS Programme of Research in South Africa, University of KwazuluNatal

Word counts: Abstract 187; Body 4607

This work was performed by the Aurum Institute for Health Research

Correspondence: BV Girdler-Brown, PO Box 59, Bergbron 1712, South Africa. Telephone +2711477 7865, Telefax +2711477 0143, e-mail hsct@icon.co.za

\section{Conflict of interest statement}

GJ Churchyard was seconded by AngloGold Ashanti to Aurum Health Research during the conduct of the study. 


\begin{abstract}
Background: The burden of silicosis, pulmonary tuberculosis and COPD is described in 624 South African goldminers 18 months after cessation of work.

Methods: This was a prevalence study. Questionnaires were administered, and spirometry, chest radiography, tuberculosis investigations, and urine HIV antibody assays were performed.
\end{abstract}

Results: Attendance was 80.1\% (624/779), mean age 49.4 years, and mean employment duration 25.6 years. Most subjects had had medium (26.5\%) or high (65.4\%) dust-exposure jobs. Current smoking rate was 35\%, with ever smoking $61 \%$. HIV antibodies were detected in the urine in $22.3 \%$. Prevalences were: silicosis $24.6 \%$, past tuberculosis $26 \%$, current tuberculosis $6.2 \%$, airflow obstruction $13.4 \%$ and chronic productive cough $17.7 \%$. Almost $50 \%$ of these miners had at least one of these respiratory conditions.

Conclusions: A heavy burden of silicosis, tuberculosis and COPD was present in this group of former goldminers. Intensification of work place dust control measures and TB and HIV prevention activities are needed on South African gold mines. In labour sending communities investment is needed in silicosis and tuberculosis surveillance as well as HIV treatment and care.

Keywords: Silicosis; Tuberculosis, Pulmonary; Pulmonary Disease, Chronic Obstructive; Human Immunodeficiency Virus; Miners 


\section{INTRODUCTION}

Gold is found in hard rock with high silica content. Chronic low level silica dust exposure predisposes to silicosis, which may present or progress years after cessation of silica dust exposure (Hnizdo and Sluis-Cremer, 1993). Both silica dust exposure in the absence of silicosis and silicosis are risk factors for tuberculosis (TB) (Zambon, 1987; Cowie, 1994; Murray and Hnizdo, 1998). Because infection with the human immunodeficiency virus (HIV) is also a strong risk factor for $\mathrm{TB}$, the risk of TB is greatly increased among miners with silicosis and HIV, in a multiplicative interaction (Corbett et al., 2000). Silica dust exposure, smoking and TB are in turn risk factors for chronic airflow limitation (Hnizdo and Vallyathan, 2003; Hnizdo et al., 2000).

In resource-limited countries relatively little is know of the burden of lung disease among former miners owing to a lack of surveillance and poor access to health services. Two studies of former black miners who worked in South African gold mines have demonstrated a high prevalence of silicosis. ("Black miners" are an occupational class of mineworkers who have historically performed the dustiest underground jobs and are largely migrant workers from rural areas of South Africa and neighbouring countries.)

Steen et al. (1997) reported a $26.6 \%$ to $31.0 \%$ prevalence of silicosis (depending on radiograph reader) in 1994 in a group of former goldminers from Botswana with average age 56.7 years, duration of mine employment 14.5 years and time since last mining work 15.7 years. Trapido et al. (1998) found a silicosis prevalence of $20.6 \%$ to $26.3 \%$ (depending on reader) in a group of former goldminers from the Eastern Cape province in 1996, with average age 52.8 years, duration of mine employment 12.2 years and time since last mine 
work 11.9 years. In addition, Churchyard et al. (2004) have reported on the prevalence of silicosis among in-service older South African goldminers. They found a prevalence of silicosis of $18.3 \%$ to $19.9 \%$ (depending on reader). In their study the mean age was 46.7 years and mean duration of service 21.8 years.

In the study by Steen et al. (1997) $29 \%$ of ex-goldminers reported that they had previously been treated for TB and $24 \%$ of chest radiographs were considered to show evidence of past and/or active TB. Physical examination by a physician resulted in the diagnosis of current active smear positive TB in $1.3 \%$ of those examined. The 1998 report by Trapido et al. gave figures of $51 \%$ for past treatment for TB and 33\% for chest radiographic changes attributable to TB. The prevalence of current active TB was not reported. Since the early 1990 s the TB case notification rate has increased significantly among in service goldminers, the increase driven largely by the HIV epidemic (Churchyard et al., 1999; Corbett et al., 2003) to more than 3000 per 100000 population per year.

The use of GOLD (Global Initiative for Chronic Obstructive Lung Disease) scores to characterise subjects' COPD is a relatively recent practice and there are as yet no published South African surveys using the GOLD classification (Workshop Report, 2005). Among the former miners from Botswana 21.9\% had evidence of airflow obstruction as indicated by a ratio of Forced Expiratory Volume in One Second to Forced Vital Capacity (FV1/FVC ratio) $<0.70 ; 43 \%$ were current smokers (Steen et al. 1997). Of the Eastern Cape former miners, 43\% had an FEV1 / FVC ratio < 0.75; 52.9\% were current smokers (Trapido et al. 1998).

At the end of March 1998 a mine shaft in the Free State province of South Africa of one gold mining company was closed for economic reasons. As a result, all 2500 miners ceased to 
work at the shaft. Seven hundred and seventy-nine of these miners from the neighbouring country of Lesotho were invited to participate in a study of the prevalence of occupationally associated lung diseases. The study was restricted to miners recruited from Lesotho as miners from Botswana and the Eastern Cape had previously been studied. Also, Basotho miners accounted for $31 \%$ of miners working at the shaft at the time of closure and it was logistically easier to follow up miners in neighbouring Lesotho.

This paper reports on the prevalence of silicosis, TB and airflow obstruction approximately 18 months after cessation of employment at a gold mine. Because of the strong association between HIV and lung disease, HIV urine antibody status is also reported. This is the first study to report on the burden of all three of these lung diseases in a group of miners within such a short time of their ceasing to work in the mines, and the first to report on GOLD scores in a group of goldminers. 


\section{MATERIALS AND METHODS}

This was a prevalence study involving 779 former goldminers. The Employment Bureau of Africa (TEBA), which has offices in all labour-sending communities, was used to locate and determine the vital status of these miners and to invite surviving miners to join the study.

Those who agreed to join the study were transported by bus to the company's mine hospital in the Free State province. These ex-miners were invited to respond to an administered respiratory history questionnaire, to submit a urine specimen for HIV antibodies, and to undergo a physical examination, a standard chest radiographic examination and spirometry.

\section{Silica dust exposure}

In order to describe the dust exposure context for this study we have classified the miners' last jobs into no dust, low dust, medium dust or high dust categories. Since there were no usable dust exposure data from this mine we made use of an existing (unpublished) dust exposure-based classification of jobs that had been carried out previously in a separate exercise on a similar operationally active gold mine.

In that exercise a company occupational hygienist categorised jobs into no, low, medium or high dust categories based on his experience and data that he had available from the mine's routine dust surveillance programme. In that population of 16383 miners the distributions between these four categories were respectively: $3.8 \% ; 26.5 \% ; 29.1 \%$; and $40.6 \%$ (G. Churchyard, personal communication, 2006). 
For the subjects included in this study their last assigned occupational code was matched with the dust exposure category allocated for the identical code used in the dust exposure exercise at the reference mine.

Last occupational codes were available for 618 of the 624 ex-miners who were seen during this study. Sixty-four different job codes were represented. Where a subject's occupational code was not represented among the reference mine categories (the case for 35 miners) the dust exposure value for a similar job was used.

\section{Respiratory questionnaire}

A modification of the American Thoracic Society respiratory questionnaire (Ferris, 1978) was translated from English into Sesotho, and then back translated by two experienced translators in order to check that the content was correctly reflected by the translation.

The questionnaire ascertained respiratory symptoms (with duration), smoking and medical history, including past or present history of treatment for TB. Trained interviewers were used to administer the questionnaire in Sesotho.

\section{HIV testing}

Testing for HIV infection was carried out using two tests on urine specimens. Specimens were first screened using the immunoglobulin $\mathrm{G}(\mathrm{IgG})$ antibody capture particle adherence test (GACPAT). Positive and equivocal results were confirmed using the $\operatorname{IgG}$ antibody capture enzyme-linked immunosorbent assay (GACELISA). 
These tests have been validated in Malawi: the sensitivity and specificity of the GACPAT test were found to be $96.5 \%$ and $98.8 \%$ respectively while those of the GACELISA were $98.8 \%$ and $99.2 \%$ respectively (Sterne et al., 1993).

\section{Chest radiography}

Standard posterior-anterior chest radiographs were taken. Radiographs were read according to the International Labour Organization (ILO) classification (International Labour Organization, 1980) by four readers. The four readers were blind to the subjects' histories as well as to each other's readings.

\section{Spirometry}

Spirometry was performed, without bronchodilation, using a Hans Rudolph pneumotachograph (Flowscan; Hans Rudolph Inc,. Kansas City MO) according to American Thoracic Society (ATS) standards as described elsewhere (American Thoracic Society, 1995).

Although bronchodilation was not performed, lung function results were classified according to the GOLD classification system (which, if correctly applied, makes use of post bronchodilation readings) (Workshop Report, 2005). For GOLD score purposes, respiratory symptoms were defined as current cough and sputum production of more than 3 months' duration. Under this classification, Grade 0 are those with an FEV1/FVC ratio $\geq 0.7$ and respiratory symptoms, ("at risk" of COPD) while the other grades require an FEV1/FVC ratio $<0.7$. 
Grade I ("mild" COPD) includes those with FEV1 $\geq 80 \%$ of their predicted value, Grade II ("moderate") $50 \% \leq$ FEV1 $<80 \%$ of predicted, Grade III ("severe") $30 \% \leq$ FEV $1<50 \%$ of predicted and Grade IV (“very severe") FEV1 < 30\% of predicted (Workshop Report, 2005). In this study, predicted values were calculated using a population specific equation derived locally from 496 non dust-exposed service workers (Louw et al., 1996).

\section{Sputum examination}

All TB suspects (based on symptoms and/or chest radiography) had two sputum specimens submitted for microscopy, culture and susceptibility. Smears were examined using fluorescent staining and specimens were cultured using Lowenstein Jensen medium. Subjects were classified as being on TB treatment if, at the time of examination, they were taking TB treatment started elsewhere (e.g. in Lesotho).

In addition, subjects who were not taking TB treatment were classified as having $\mathrm{TB}$ and were started on TB treatment if they had bacteriological proof of disease (either on sputum smear examination or culture) or on clinical grounds.

\section{Estimation of the pulmonary tuberculosis treatment incidence rate}

Assuming a steady state, the annual rate at which TB treatment is started may be estimated from the prevalence of individuals on TB treatment and the duration of treatment (incidence $=$ prevalence on treatment/mean duration of treatment). We assumed for the purposes of calculation that those taking TB treatment without a previous history of tuberculosis were taking a six-month course of treatment and that those with a previous history were on an eight-month course of treatment. 


\section{Death of a study participant}

Personnel were sent out to look for all study recruits who did not arrive for their medical examinations. Where relatives and community members reported that an ex-miner had died the cause, date and place of death was established from relatives using a modified verbal autopsy instrument (Chandramohan et al., 1998).

\section{Statistical analysis}

Data were double entered into MS Access and then exported to Stata version 7. Weighted and unweighted kappa statistics were estimated for inter-rater agreement on chest radiograph readings. The system proposed by Byrt (1996) was used to interpret the kappa statistic. The silicosis readings of the reader with the best average kappa statistics were used for the estimation of silicosis prevalence.

\section{Ethics}

The study protocol was approved by the Research Ethics Committee of the Faculty of Health Sciences at the University of Cape Town and by the AngloGold Health Services medical research ethics committee. Informed consent was obtained in writing from all participants.

HIV test results were provided to the researchers by study number only, and so full anonymity of the individuals was preserved. Subjects who wished to know their HIV status were referred to the company's HIV confidential voluntary counselling and testing services where the test was repeated with pre- and post-test counselling. Individuals diagnosed with TB were started on TB treatment and, if required, hospitalized until well enough to be discharged. TB patients were given a referral letter to their local clinic in Lesotho to continue TB treatment. 


\section{RESULTS}

Six hundred and twenty-four of the 779 former miners from Lesotho $(80.1 \%)$ were seen. The mean interval between the date of work cessation and the examination was 1.5 years (range 1.4 to 1.8 years). The characteristics of those who attended are summarised in Table I.

Among the current smokers, the median number of cigarettes smoked per day was four (range 2- 20). In addition there were ten who smoked pipes only (between twice and seven times a day with a mean of 3.6). Distribution of dust exposure categories is illustrated in Figure 1.

\section{Deaths between ceasing to work on the mine and the study survey}

Forty-one out of 155 of the ex-miners who were included in the study but who did not report for their examination were found to have died since leaving the mine. Thirty-five out of the $41(85.4 \%)$ had symptoms of lung disease (e.g. coughing or coughing up blood) before death. The other six were reported to have died from assaults (two) and one each of "accident", "myocardial infarction" "severe diarrhoea" and "cancer of the oesophagus".

The prevalence of HIV infection among those who died could not be ascertained. The mean age (as of 22 March 1998, the median date of last employment) of those who died was greater than that of those who survived by 1.85 years (49.2 and 47.4 years respectively, $\mathrm{p}=0.02$ ).

\section{Silicosis}

The weighted kappa statistics presented in Table II varied from 0.58 to 0.74 using the more conservative weighting system (option 1) and demonstrate "good" agreement according to 
Byrt's guidelines. Reader 3 had the best overall agreement. Using an ILO score of $\geq 1 / 1$ as indicating definite silicosis and reader 3's readings, the prevalence of silicosis was $24.6 \%$.

\section{Pulmonary tuberculosis}

Twenty-six percent of the 624 subjects for whom data was available (165/624) had a past history of TB and $1.3 \%(8 / 624)$ were already on TB treatment at the time of the study examination: of these eight, four had a past history of TB. Assuming that those with a history of past TB were on an 8-month retreatment regimen while the remainder were on a 6-month treatment regimen, these findings are compatible with an annual incidence rate for being placed on TB treatment of 2198 cases per 100000 per year.

Of the remaining 616 subjects who were not on TB treatment, 18 (2.9\% of the 624$)$ had active undiagnosed TB confirmed on microscopy or culture. A further $13(2.1 \%$ of the 624$)$ were started on TB treatment on clinical grounds.

Of the eight subjects who were already taking $\mathrm{TB}$ treatment at the time of the study examination, three were HIV positive, three HIV negative and the HIV status was unknown for the other two. Of the 31 subjects who were diagnosed with TB and placed on treatment at the time of the study examination, $8 / 31$ were HIV positive, 22/31 were HIV negative and the HIV antibody status was unknown in one case.

\section{Airflow obstruction}

The FEV1/FVC ratios were calculated for 620 miners for whom data were available. One hundred and eight of these 620 miners (17.7\%) had cough and sputum for at least 3 months duration. The FEV1/FVC ratio was less than 0.7 in $13.5 \%$ (and less than 0.75 in $26.3 \%$ ) of 
these ex-miners. The lung function tests and current symptoms of cough and sputum $(>3$ months) were classified in the 610 subjects for whom all data were available according to the GOLD classification for obstructive airways disease. These results are presented in Table III.

\section{Total burden of silicosis, tuberculosis and COPD}

Figure 2 illustrates the total prevalent burden of silicosis, current or past TB and airflow obstruction (GOLD score Grade I or higher) taking into account that some subjects had more than one of the conditions, and that GOLD scores were assigned without bronchodilation prior to spirometry. Only 610 subjects are included since full information was not available for 14 of the 624 ex-miners who attended for examination.

Forty-nine point five percent of these 610 ex-miners had one or more of silicosis, airflow obstruction or a history of TB (past and/or current). The percentage with past and/or current TB was $30.2 \%$. 


\section{DISCUSSION}

This study shows a high prevalence of silicosis, TB and airflow obstruction in a group of Basotho mine workers 18 months after their employment at a South African gold mine was terminated on economic grounds.

Almost $25 \%$ had silicosis (grade $1 / 1$ or greater), $13.9 \%$ had at least "mild" airflow obstruction, $26 \%$ had a past history of $\mathrm{TB}, 1.28 \%$ were taking treatment for $\mathrm{TB}, 2.9 \%$ had bacteriologically proven TB and were started on treatment for TB and $2.1 \%$ were started on TB treatment on clinical grounds. In addition, $85.4 \%$ of those who died after the end of their employment at the mine had respiratory symptoms on verbal autopsy.

This study was prospectively planned and achieved a good response rate of $80.1 \%$. The data for this study were collected under research conditions and reliance was not placed on routinely collected data. Furthermore, HIV antibody status was ascertained for nearly all participants and verbal autopsy was performed for those who were known to have died. It is unlikely that all deaths were identified and some of the 114 miners who did not attend, and who were not recorded by us as having died, may in fact have died.

There are a number of considerations affecting the representativeness of the dust exposure of this group and thus generalisability of the findings to the goldmining industry as a whole. It was not possible to obtain respirable dust measurements from personal dosimetry records for the study participants, so we have had to rely on a classification of their exposures into "high", "medium" and "low" based on their last job codes using qualitative assessments derived from another mine. The average duration of employment at the mine was 25.6 years 
and it is likely that at least some of the participants had previously been employed in different jobs (with different dust exposures) during this time. It is also possible that over time some of the employees of this mine might have elected to move to less dusty work, or even to seek work elsewhere as a result of respiratory symptoms and therefore not have been available for this study.

Almost $92 \%$ of the miners were working in medium or high dust exposure jobs immediately prior to the closure of the shaft. In comparison, only $70 \%$ of all currently employed miners at the reference gold mine were in a medium or high dust exposure job. This difference may have occurred due to differential job loss in the months prior to closure of the mine studied, with production jobs (which are medium to high dust exposure occupations) being preferentially retained until closure of the mine. Another potential source of selection bias in this regard is the selection into the study only of men originating from Lesotho. Basotho men are known in the industry to be overrepresented in physically demanding production jobs, which may have resulted in a greater proportion of men being highly dust-exposed in this study than in the industry as a whole.

Previous studies of surviving former goldminers living in the Libode district (Trapido et al., 1998) of the Eastern Cape and in the Tamaga district (Steen et al., 1997) in Botswana reported prevalences of silicosis of $20 \%$ to $30 \%$. These men had left mining employment on average 12 to 15 years previously.

A limitation of these two studies is that they included only surviving former miners who were resident in the sending areas at the time of study. As a result of this limitation the prevalence of silicosis may have been underestimated if those most seriously affected by silicosis had 
died during the 12 to 15 years since last mining employment. Conversely, silicosis prevalence may have been overestimated if a substantial proportion of healthy miners from those areas were excluded because they had found alternative employment and were not resident in the area at the time of the study.

However, in a representative sample of in-service (older) goldminers employed by the same company as in this study but at a different shaft, Churchyard et al. (2004) found a silicosis prevalence of as high as $18 \%$ to $20 \%$. In our study the mean duration of service was almost four years longer (25.6 vs. 21.9 years) and in addition there were 18 months of "residence time" during which silicosis could have progressed even in the absence of further exposure to dust containing crystalline silica.

The prevalence of silicosis $(24 \%)$ reported in this study is thus of a similar order of magnitude to those found in these earlier studies. However, because miners with severe silicosis might be declared medically unfit for work or choose not to continue with mining work, cross-sectional studies are always likely to provide us with a somewhat censored idea of the real lifetime risk that is faced by underground miners employed in South African gold mines.

The high rates of TB observed in this study may be due to several possible factors, not all of which were investigated in this study. We have documented high rates of both HIV infection (22.3\% had urine antibodies) and silicosis among the study subjects. These are two powerful risk factors for $\mathrm{TB}$, whose combined effect has been shown to be multiplicative (Corbett et al., 2000). Even in the absence of radiographic evidence of silicosis, studies reported by Cowie (1994), Murray and Hnizdo (1998) and te Water Naude et al. (2006) have shown that 
the risk of TB is increased by exposure to dust containing crystalline silica. Other possible risk factors for TB include working and living arrangements on the mine predisposing to an increased rate of transmission of the tubercle bacillus and high rates of transmission in the miners' home communities in Lesotho.

It is noteworthy that $6.28 \%$ of these miners were either taking treatment for $\mathrm{TB}$ started elsewhere $(1.28 \%)$ or else were started on anti-TB treatment after being seen during the study (5\%). Almost 3 out of 5 of the latter group had bacteriologically proven TB. Since some of those taking ant-TB treatment that was started elsewhere (e.g. in Lesotho) might have been too ill to travel or might have died from their disease the estimate of TB incidence obtained in this study would underestimate the true incidence rate of TB in these miners.

At the time that the shaft was closed all the miners had an exit medical examination that included a TB symptoms screening questionnaire and a chest radiograph for TB. If TB was suspected the men were first investigated for TB before returning home. It is known that some miners returned home without waiting for the results of these investigations, and it is possible that some of the miners who were found to have active TB during the study might have had their TB disease since the time that the shaft was closed.

The high proportion (almost 3\%) of these miners who had bacteriologically proven TB suggests either an asymptomatic stage of the disease or failure to access TB diagnostic and treatment services in their home communities. Since $1.28 \%$ of the miners were on treatment for TB when they were seen, and assuming that these all had bacteriologically proven TB, approximately $70 \%$ of those with bacteriologically demonstrable active TB were not on treatment for their disease. 
Spirometry was carried out in this study without bronchodilation. As a result the prevalence of airflow obstruction (13.93\% with GOLD score $>0)$ may have included some cases of asthma rather than COPD and would therefore be an overestimate of COPD. Of this figure, $5.7 \%$ were "mild" (FEV1> 80\% predicted), $6.5 \%$ moderate and only $1.2 \%$ severe.

The overall prevalence of COPD is a little higher than a "global average" estimate of physiologically defined COPD in adults over 40 years of 9-10\% (Halbert et al., 2006) but lower than that found in the two studies of ex-miners cited earlier (Steen et al., 1997, Trapido et al., 1998). The proportion of miners with chronic cough and sputum for three months or longer $(17.7 \%)$ was considerably higher (although on a less strict definition) than the prevalence of chronic bronchitis (which requires the presence of these symptoms for at least three months in at least two successive years) in the general South African male population aged $45-54$ years of $4.3 \%$ (Ehrlich et al, 2004), and in the middle range of prevalence found in a number of occupational populations in Africa exposed to dust (Chan-Yeung et al., 2004).

Although cumulative dust exposures could not be calculated, the long average duration of service (25.6 years) and the high proportion of the miners in dusty jobs at the end of their service on the mine make it likely that cumulative exposures were high. In addition, $26 \%$ had a past history of TB. As a result we would have expected a higher prevalence of airflow obstruction, and of cough and sputum. Previous studies have shown an interaction between tobacco smoking and dust in producing clinically significant COPD (Hnizdo, 1992). In this workforce the median number of cigarettes was relatively low despite $34.8 \%$ being current smokers. In addition, miners may have better lung function at work entry than the general 
population (Hnizdo, 1992) while symptoms may select workers out of the dusty mining jobs, creating a "healthy survivor" effect in this workforce.

These high rates of lung disease among former goldminers are of national concern since silicosis, TB, COPD, and HIV infection (an important predisposing cause for TB) are all preventable conditions. Chronic silicotuberculosis, COPD and HIV infection in turn produce a huge burden of morbidity and health care need. Access to diagnostic, treatment and compensation services is known to be poor in many labour sending communities, compounding the serious health and economic consequences of lung disease for former miners, their families and communities.

These findings, and their consistency with previously published reports on the prevalence of respiratory disease in ex-miners, provide a compelling case for improved statutory surveillance and reporting of silicosis and TB among former goldminers, not just for compensation purposes, but also in order to monitor the effectiveness of control measures aimed at reducing the risk of silicosis TB and COPD.

The reduction of silica dust exposure is of great importance: first to reduce the risk of silicosis and second to reduce the risk of TB. The occupational exposure limit for respirable silica that would minimise the risk of silica associated TB is unknown. However, since both silica dust and silicosis increase the risk of pulmonary $\mathrm{TB}$, reduction of silica exposure and prevention of new cases of silicosis would be a necessary step in reducing this risk (Churchyard et al., 2004). 
In South Africa, silica dust exposure in the mines is routinely monitored by means of periodical personal dosimetry. The results have to be reported to the national Department of Minerals and Energy. At the time that this study was carried out the time weighted average that was deemed acceptable for respirable crystalline silica exposure was (and still is) 0.1 $\mathrm{mg} / \mathrm{m}^{3}$ for miners. Although there has been a national tuberculosis control programme in place in South Africa for more than 20 years, the national silicosis elimination programme was only launched more recently and was not in force at the time that these miners were working at the (now) closed shaft. Dust control measures in turn have to be accompanied by comprehensive TB and HIV prevention activities among in service miners.

Finally, investment in health systems in rural labour sending communities is needed. A comprehensive programme for former miners would include surveillance to detect occupationally associated lung diseases, as a check on control measures on the mines and for compensation purposes, and provision of treatment, care and support for those diagnosed with these conditions. 


\section{ACKNOWLEDGEMENTS}

We are grateful to the former miners who took part in the study and to the National Union of Mineworkers for its support for the study. The following are thanked individually for their participation: R de Bois (Study manager); D Banks and J Parker (additional radiograph readers). AngloGold Corporation is thanked for funding this study and for giving prior permission to publish the results irrespective of the outcome. We acknowledge the contribution of our co-author Professor Neil White, who died before the report was completed, to occupational health in South Africa and particularly to protecting the health of mine workers. 
Table I. Demographic characteristics and risk factors for silicosis, pulmonary tuberculosis and COPD among former goldminers

\begin{tabular}{lcc}
\hline Characteristic & N* & Mean (range) or \% \\
\hline Age (years) & 623 & $49.4(25.9$ to 61.7) \\
Duration of employment (years) & 617 & $25.6(0.9$ to 40.2$)$ \\
Medium dust work during last job (\%) & 624 & 26.2 \\
High dust work during last job (\%) & 624 & 65.3 \\
Current smoker (\%) & 624 & 34.8 \\
Ever smoked (\%) & 624 & 61.4 \\
HIV antibodies positive (\%) & 578 & 22.3 \\
Past pulmonary tuberculosis (\%)** & 624 & 26.4 \\
\hline
\end{tabular}

*The value of $\mathrm{N}$ varies according to the number who had information available

** Self-reported.

Table II. Kappa statistics for inter-rater agreement in reading chest radiographs for silicosis using the ILO Classification $\left(\mathrm{N}=619^{*}\right)$

\begin{tabular}{lcccc}
\hline Comparison** & \% agreement & $\begin{array}{c}\text { Unweighted } \\
\text { kappa }\end{array}$ & $\begin{array}{c}\text { Weighted } \\
\text { (option 1) }\end{array}$ & $\begin{array}{l}\text { Weighted }{ }^{\mathrm{b}} \text { kappa } \\
\text { (option 2) }\end{array}$ \\
\hline R1 R2 & 57.8 & 0.3078 & 0.5858 & 0.7499 \\
R1 R3 & 97.3 & 0.7499 & 0.5849 & 0.7393 \\
R1 R4 & 57.8 & 0.3038 & 0.5835 & 0.7353 \\
R2 R3 & 97.0 & 0.7353 & 0.6232 & 0.7724 \\
R2 R4 & 65.9 & 0.3668 & 0.6242 & 0.7618 \\
R3 R4 & 69.1 & 0.4730 & 0.7354 & 0.8443 \\
\hline
\end{tabular}

*This includes only those miners who had their chest radiographs read and commented upon by all 4 readers.

** R1R2 refers to reader 1 and reader 2, R1R3 refers to reader 1 and reader 3, etc...

a Weights $1-\mid \mathrm{i}-\mathrm{j} / /(\mathrm{k}-1)$, where $\mathrm{i}$ and $\mathrm{j}$ index the rows and columns of the ratings by the two raters and $\mathrm{k}$ is the maximum number of possible ratings

b Weights $1-[(\mathrm{i}-\mathrm{j}) /(\mathrm{k}-1)]^{\wedge} 2$, where $\mathrm{i}$ and $\mathrm{j}$ index the rows and columns of the ratings by the two raters and $\mathrm{k}$ is the maximum number of possible ratings 
Table III. Airflow obstruction among former goldminers $\left(\mathrm{N}=610^{*}\right)$ by GOLD** score

\begin{tabular}{lrrrrrr}
\hline GOLD SCORE & Nil & $0^{* * * *}$ & I & II & III & IV \\
\hline $\mathrm{N}$ & 443 & 85 & 35 & 40 & 7 & 0 \\
$\%$ & 72.6 & 13.9 & 5.7 & 6.6 & 1.2 & 0.0 \\
Cumulative \% & 72.6 & 86.6 & 92.3 & 98.9 & 100.0 & 100.0
\end{tabular}

*This includes only those 610 miners for whom a full set of information (spirometry results and a recorded history of respiratory symptoms) was available.

** The GOLD score is the scoring system for obstructive lung disease that has been described by the Global Initiative for Chronic Obstructive Lung Disease (Workshop report 2005)

*** Subjects who are scored as "Nil" and " 0 " all have FEV1/FVC ratios of 0.7 or greater. The difference between the two is that those scored as " 0 " have chronic respiratory symptoms, defined in this study as cough with sputum for at least three months in duration.

Figure 1. Percentage distribution of dust exposure categories assigned to jobs of the participants alongside those of the reference mine

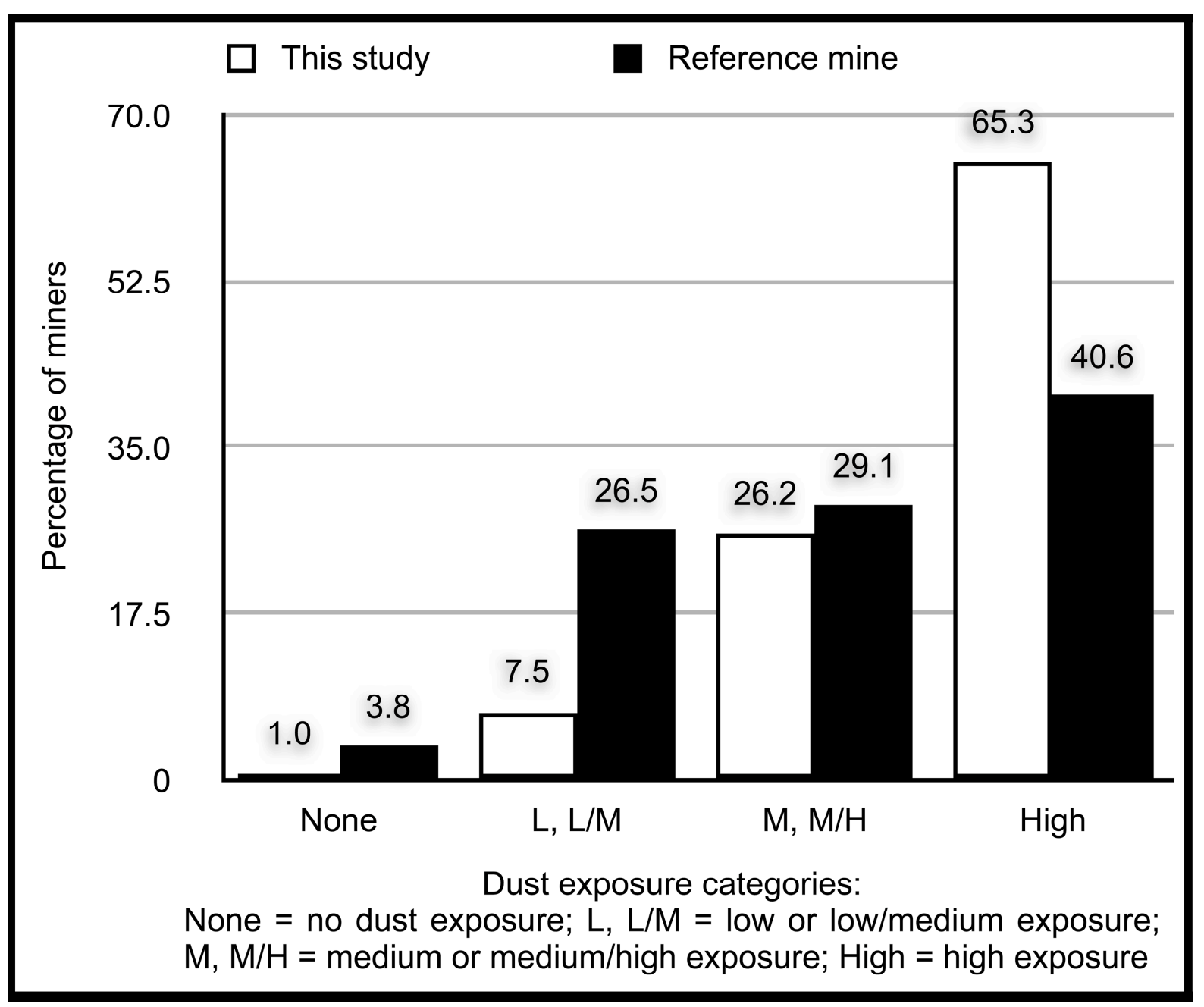


Figure 2. Numbers and percentages of former goldminers $(\mathrm{N}=610)$ with silicosis, airflow obstruction and current or past TB

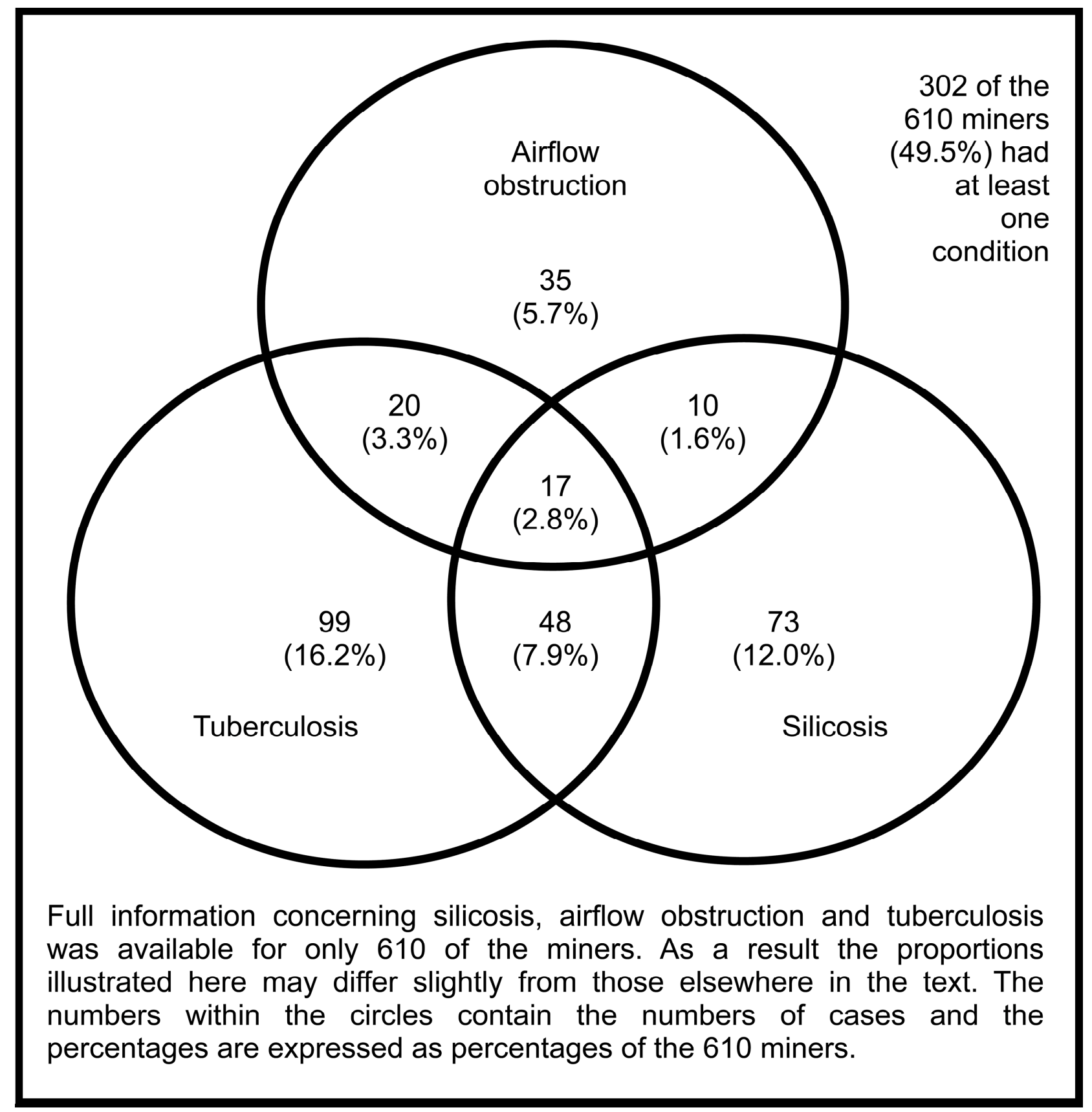




\section{REFERENCES}

American Thoracic Society. 1995. Stardardization of spirometry - 1994 update. Am J Respir Crit Care Med 152:1107-36.

Byrt T. 1996. How good is that agreement? Epidemiol 7:561.

Chandramohan D, Maude GH, Rodrigues LC, Hayes RJ. 1998. Verbal autopsies for adult deaths: their development and validation in a multicentre study. Trop Med Int Health 3:436-46.

Chan-Yeung M, Ait-Khaled N, White N, Ip MS, Tan WC. 2004. The burden and impact of COPD in Asia and Africa. Int J Tuberc Lung Dis 8:2-14.

Churchyard GJ, Kleinschmidt I, Corbett EL, Mulder D, De Cock KM. 1999. Mycobacterial disease in South African gold miners in the era of HIV infection. Int J Tuberc Lung Dis 3:791-8.

Churchyard GJ, Ehrlich RI, te Water Naude JM, Pemba L, Dekker K, Vermeijs M, White N, Myers J. 2004. Silicosis prevalence and exposure-response relations in South African goldminers. Occup Environ Med 61:811-6.

Corbett EL, Charalambous S, Fielding K, Clayton TC, Hayes RJ, De Cock KM, Churchyard GJ. 2003. Stable tuberculosis incidence rates of tuberculosis (TB) among human immunodeficiency virus (HIV)-negative South African gold miners during a decade of epidemic HIV-associated TB. J Infect Dis $15: 1156-63$.

Corbett EL, Churchyard GJ, Clayton TC, Williams BG, Mulder D, Hayes RJ, De Cock KM. 2000. HIV infection and silicosis: The impact of two potent risk factors on incidence of mycobacterial disease in South African miners. AIDS 14:2759-68.

Cowie RL. 1994. The epidemiology of tuberculosis in gold miners with silicosis. Am J Respir Crit Care Med 150:1460-2.

Ehrlich RI, Norman R, Laubscher R, White N, Steyn K, Lombard C, Bradshaw D. 2004. Predictors of chronic bronchitis in adults in South Africa. Int J Tuberc Lung Dis 8:369-76.

Ferris BG. 1978. Epidemiology Standardization Project. Am Rev Respir Dis 118:1-113.

Halbert RJ, Natoli JL, Gano A, Badamgarav E, Buist AS, Mannino DM. 2006. Global burden of COPD: systematic review and meta-analysis. Eur Respir J 28:523-32.

Hnizdo E. 1992. Health risks among white South African goldminers - dust, smoking and chronic obstructive pulmonary disease. S Afr Med J 81:512-7.

Hnizdo E, Singh T, Churchyard GJ. 2000. Chronic pulmonary function impairment caused by initial and recurrent pulmonary tuberculosis following treatment. Thorax 55:32-8. 
Hnizdo E, Sluis-Cremer G. 1993. Risk of silicosis in a cohort of white South African gold miners. Am J Ind Med 24:447-57.

Hnizdo E, Vallyathan V. 2003. Chronic obstructive pulmonary disease due to occupational exposure to silica dust: a review of epidemiological and pathological evidence. Occup Environ Med 60:237-43. International Labour Organization. 1980. Guidelines for the Use of ILO International Classification of Radiographs of Pneumoconioses. Occupational Safety and Health Series No. 22. Geneva: ILO.

Louw SJ, Goldin JG, Joubert G. 1996. Spirometry of healthy adult South African men. Part I. Normative values. S Afr Med J 86:814-9.

Murray J, Hnizdo E. 1998. Risk of pulmonary tuberculosis relative to silicosis and exposure to silica dust in South African gold miners. Occup Environ Med 55:496-502.

Steen TW, Gie KM, White NW, Gabosianelwe T, Ludick S, Mazonde GN, Mabongo N, Ncube M, Monare N, Ehrlich R, Schierhout G. 1997. Prevalence of occupational lung disease among Botswana men formerly employed in the South African mining industry. Occup Environ Med 54:19-26.

Sterne JA, Turner AC, Connell JA, Parry JV, Fine PE, Ponnighaus JM, Nyasuku S, Mkandwire PK. 1993. Human immunodeficiency virus: GACPAT and GACELISA as diagnostic tests for antibodies in urine. Trans R Soc Trop Med Hyg 87: 181-3.

Te Water Naude JM, Ehrlich RI, Churchyard GJ, Pemba L, Dekker K, Vermeijs M, White NM, Thompson ML, Myers JE. 2006. Tuberculosis and silica exposure in South African gold miners. Occ Environ Med 63:187-92.

Trapido AS, Mqoqi NP, Williams BG, White NW, Solomon A, Goode RH, Macheke CM, Davies AJ, Panter C. 1998. Prevalence of occupational lung disease in a random sample of former mineworkers, Libode District, Eastern Cape Province, South Africa. Am J Ind Med 34:305-13.

Workshop Report: Global Strategy for the Diagnosis, Management, and Prevention of COPD Updated 2005. 2005. Available from http:// www.goldcopd.org

Zambon P. 1987. Mortality of workers compensated for silicosis during the period 1959-1983 in the Veneto Region of Italy. Scand J Work Environ Health 13:118-23. 